\title{
Semantic priming: Subliminal perception or context?
}

\author{
IRA H. BERNSTEIN, VICTOR BISSONNETTE, AVNI VYAS, and PAMELA BARCLAY \\ University of Texas at Arlington, Arlington, Texas
}

\begin{abstract}
Briefly presented, masked priming stimuli that cannot be identified by themselves can affect the processing of subsequent targets. The effect, which is sometimes viewed as a demonstration of unconscious processing, has been linked to the subliminal perception literature. Experiments 1 and 2 indicated that the identification of primes in the context of semantically related targets is superior to the identification of primes presented alone, and that the identification of primes in the context of semantically unrelated targets is inferior to the identification of primes presented alone. Experiment 3 indicated parallel findings in a recognition task. Consequently, an explanation of semantic priming in terms of the interactive nature of stimuli that are near to one another in time seems preferable to one based upon concepts of unconscious processing and subliminal perception.
\end{abstract}

Marcel (1980, 1983a, 1983b) first reported a form of semantic priming that involved briefly presented, masked priming stimuli. The essentials of his effect are that the primes cannot be identified when presented alone, but that they modify responses to semantically related, supraliminal target stimuli. The effect is presumed to illustrate "subliminal" effects of masked stimuli, in that the primes operate "unconsciously," "automatically," or "without awareness."

For example, suppose that the words DOG and CHAIR are masked and presented so briefly that they cannot be identified. Now, present the sequence DOG/LION on one trial and the sequence CHAIR/LION on another trial. DOG and CHAIR are presented as before, but LION is clearly visible. The subjects' task in both cases is to say "LION" as quickly as possible. Marcel's effect will be illustrated if the naming of LION occurs more rapidly when it is preceded by a fellow member of the animal category, DOG, as opposed to an unrelated word such as CHAIR (additional pairings are of course necessary in order to control for the difference in length between DOG and CHAIR, etc.). Carr, McCauley, Sperber, and Parmelee (1982), Fowler, Wolford, Slade, and Tessinary (1981), Hines, Czerwinski, Sawyer, and Dwyer (1986), and McCauley, Parmelee, Sperber, and Carr (1980) have replicated this effect in various ways. Fowler et al. (1981) and Marcel (1980), for example, used lexical decisions, whereas Hines et al. (1986) and McCauley et al. (1980) used naming latencies.

Both Merikle (1982) and Purcell, Stewart, and Stanovich (1983) have suggested that this effect is spuri-

A portion of these results was presented at the 1987 meeting of the Psychonomic Society, Seattle, WA. The authors are grateful to Heidi Brown, Laura Torbett, and Robert Boyd for their assistance in gathering data, and to Roger Schvaneveldt and Nikki Shute for their comments. Requests for reprints should be addressed to Ira H. Bernstein, Department of Psychology, University of Texas at Arlington, P.O. Box 19528, Arlington, TX 76019-0528. ous. Fowler et al. (1981) had concluded that their own primes were subliminal because their subjects could not discriminate primes followed by a mask from blank fields followed by a mask. Merikle (1982) showed that this failure could simply reflect the adoption of a very stringent criterion for reporting the presence of primes. More recently, Merikle's theoretical position has changed somewhat (see Cheesman \& Merikle, 1984, 1986), but his previous criticisms are still applicable. His more recent work will be considered later in this paper.

Purcell et al. (1983) argued that the subjects' sensory adaptation on threshold determination (prime + mask) trials is different from their sensory adaptation on semantic priming (prime + mask + target) trials. In effect, the mask is weaker in the latter case, making the prime more visible. Holender (1986) has provided a lengthy critical summary of this literature.

We suggest that although Merikle's (1982) and Purcell et al.'s (1983) points are well taken, semantic priming effects could, in principle, be obtained in studies free of the above cited artifacts. Even so, we hope to show that it has nothing to do with "unconscious" or "automatic" processing, "subliminal perception," or related concepts. We suggest that it has everything to do with cues furnished by targets that aid prime identification and recognition. Our hypothesis, therefore, is that targets provide information about the primes, and vice versa.

It should not be surprising that a word could be presented so briefly that its chances of being identified are immeasurably low, yet identified when appropriately cued. Assume that a word is flashed so briefly that a subject refuses to hazard a guess.

Suppose, however, that the subject is fairly certain that the word had six letters and that the two middle letters could be LL or II (or perhaps LI or IL). Even so, the range of possibilities will clearly be quite great, and the incentive for guessing will be sufficiently limited to lead the experimenter to believe that the subject, who has not ven- 
tured a guess, has not seen the word. Now, assume the words TEXAS CITY appear clearly afterwards. This information will make a correct response like "DALLAS" much more probable.

The preceding example involves the interaction of an impoverished stimulus and a subsequent, clearly visible event. There is a long history of similarly illustrative studies. These studies generally indicate that relevant information provided by the second event improves judgments about the first one. Some of the more classical studies, including those using auditory presentation, include Lawrence and Coles (1954), Pollack, Rubenstein, and Decker (1959), Rubenstein and Pollack (1963), Swets and Sewall (1963), and-perhaps the most widely cited of all-Sperling (1960).

This earlier literature subsumes a wide range of phenomena and issues. For our present purposes, it is sufficient to note that all of these studies illustrate the effectiveness of poststimulus cuing. Moreover, still other studies illustrate how context in general can affect the accuracy of judging semantic material (e.g., Pierce, 1963; Tulving \& Gold, 1963).

The major difference between priming studies and poststimulus cuing studies is that priming studies focus upon the more, rather than the less, intense event. In other words, what contemporary investigators refer to as primes and targets have historically constituted discriminanda and cues. This does not seem adequate reason for the stronger event not to influence the weaker.

Related literature and our hypothetical "study" illustrate that information possessed by the subject is much more complex than the summary data contained on the experimenters' score sheets would indicate (see Broadbent, 1967; Eriksen, 1960; Natsoulas, 1967). Consequently, it is hazardous to identify "unconsciousness" with a failure of identification. Unfortunately, how to assess the entire contents of a subject's consciousness remains an unresolved problem.

Previous research has also illustrated how the processing of logically independent events may be mutually facilitative. For example, Meyer and Schvaneveldt (1971) presented pairs of letter strings. In one experiment, subjects responded "yes" if both strings were words, and "no" if either string was a nonword. They found that "yes" responses to related word pairs occurred much faster than "yes" responses to unrelated word pairs. Analogous results were found in a second experiment, in which the subjects responded "same" if both strings were words or if both strings were nonwords, and "different" if one string was a word and the second string was a nonword. The difference between their study and a semantic priming study was that both strings were presented briefly. In semantic priming experiments of the type presently considered, of course, targets are at a much higher energy level than primes. Their results would also seem more consistent with our nonsubliminal interpretation than with a "subliminal" interpretation, although proponents of the latter view frequently cite it.
The "subliminal" nature of primes is inferred from trials in which the target is absent; there is relatively little data available about how identifiable primes are on prime + mask + target trials (see below). Yet, the various early studies we have cited suggest that it is essential to determine what information targets provide to primes.

\section{EXPERIMENTS 1 AND 2}

Basically, the first two studies to be presented here employed Hines et al.'s (1986, Experiment 1) procedures, with the one major difference being that our interest was limited to the identification of the primes. Because we only attempted to replicate their first experiment, all subsequent procedural references should be taken as referring to this study. We never evaluated target responses since we assume that they may be facilitated by semantically related primes. Hines et al.'s critical finding involved an interaction between the semantic relatedness of prime/target pairings and the position of the words within the semantic categories they used. The targets were named faster when preceded by a related first exemplar prime as opposed to an unrelated first exemplar prime, but there was no such difference for the naming of targets preceded by fifth exemplar primes. Thus, DOG (the first exemplar of the "animals" category) facilitated the naming of LION (the fifth exemplar of the "animals" category) compared with the control sequence CHAIR/LION, but LION did not facilitate the naming of DOG. Knowledge of the pairs, provided by pretraining, had no effect.

\section{Method}

Subjects. Two separate groups of 30 undergraduates served in two separate experiments and received course credit for participation. The two studies were conducted sequentially.

Stimuli and Apparatus. The stimulus list, which was the same as that employed by Hines et al. (1986), consisted of the first and fifth exemplar from each of 12 normed categories (Battig \& Montague, 1969). Our procedures followed those of Hines et al. (1986) in that the sixth exemplar was used in two cases where the fifth exemplar was too long. Except as noted, we also followed their apparatus, procedures, and design in all other respects.

The stimuli were displayed on a 12-in. Zenith Data Systems 40 column monitor, controlled by an Apple Ile microcomputer. Each uppercase letter, $6 \mathrm{~mm}$ tall, subtended a visual angle of $21^{\prime}$ at the viewing distance of $99 \mathrm{~cm}$ used in Experiment 1, and 18' at the viewing distance of $114 \mathrm{~cm}$ used in Experiment 2. A five-letter word subtended $1^{\circ} 6^{\prime}$ in Experiment 1 and $57^{\prime}$ in Experiment 2. The luminance of the green letters was $100 \mathrm{~cd} / \mathrm{m}^{2}$ in Experiment 1 and $50 \mathrm{~cd} / \mathrm{m}^{2}$ in Experiment 2.

The stimuli were centered on the screen so that primes, masks, and targets overlapped. Hines et al. (1986) used reverse video, but we did not. Hines et al. did not report the stimulus characteristics of their display, which was a cathode-ray tube driven by a Timex computer, except to note that their letters were also centered and upper case. Presentation timing was accomplished with Diener and Smee's (1984) subroutines.

Experimental design. Half the subjects were assigned to the knowledge group. They first memorized the word pairs, attempted to identify masked primes in the first prime + mask condition, attempted to identify masked primes when followed by targets in the prime + mask + target condition, and, finally, attempted again to 
identify masked primes presented alone in the second prime + mask condition. The remainder were assigned to the no-knowledge group. They participated in a filler task in lieu of memorization, but did participate in the remaining three conditions.

Learning of related pairs. Subjects in the knowledge condition were read a category name aloud, followed by a pair of words reflecting that category. The experimenter proceeded twice through the list of 12 categories and associated word pairs within each category. Next, each category name was read, and the subject attempted to name the exemplars in that category. This was repeated until the subject made no mistakes.

Initial prime + mask trials. Subjects were adapted for $5 \mathrm{~min}$ to a mesopic level; the ambient illumination provided $2 \mathrm{~cd} / \mathrm{m}^{2}$ on an $18 \%$ reflectance gray card. This level of illumination remained constant throughout the experiment. We used a modified staircase approach to determine an initial threshold. The sequence consisted of a 900 -msec fixation cross, a 100 -msec blank screen, a variable duration prime, and a 200 -msec mask consisting of the letters "QZQZQZQZQZ." Presentation time for the prime began at $250 \mathrm{msec}$; it was reduced by $16.7 \mathrm{msec}$ (one screen refresh cycle) for each correct response. The threshold was defined when a subject made four consecutive incorrect responses at a given duration, or when the minimum presentation time of $33.3 \mathrm{msec}$ was reached. Words used in this portion of the experiment were not used elsewhere.

Prime + mask + target trials. The prime + mask + target trials were like prime + mask trials, except that the fixation cross reappeared after the mask for $300 \mathrm{msec}$, the screen was blank for $100 \mathrm{msec}$, and the target appeared for $300 \mathrm{msec}$, as in Hines et al. (1986). ${ }^{1}$ The interstimulus interval separating prime offset from target onset was therefore $900 \mathrm{msec}$.

In Experiment 1, the subjects were simply asked to name the prime. In Experiment 2, the subjects were instructed to name the target as quickly as possible by speaking into a microphone, which they were told was connected to a voice key. Following their response to the target, they were then asked to name the prime (a procedure employed by Carr et al., 1982). In actuality, no voice key was employed, and the subjects were debriefed about the deception at the end of the study.

Consequently, the subjects in Experiment 1 were led to believe that the experiment concerned the perception of primes, but the subjects in Experiment 2, like those in a conventional semantic priming study, focused upon response to the target. Experiment 2 was conducted to make prime identification incidental to target identification, as it is in a conventional priming study.

Following five practice trials with words not otherwise used in the study, 48 scored trials were run, 12 of each of four types: (1) the prime was the first exemplar from a given category, and the target was the fifth exemplar from that same category (e.g., DOG/LION), (2) the prime was the fifth exemplar from a given category, and the target was the first exemplar from that same category (e.g., LION/DOG), (3) the prime was the first exemplar from a given category, and the target was the fifth exemplar from a different category (e.g., DOG/DESK), and (4) the prime was the fifth exemplar from a given category, and the target was the first exemplar from a different category (e.g., LION/CHAIR). Each specific word, such as DOG, appeared four times: as a related prime, an unrelated prime, a related target, and an unrelated target. On unrelated trials, first exemplars were randomly paired with fifth exemplars. The 48 word pairs were run in a different random order for each subject.

Three within-subject variables were: first exemplar as prime/fifth exemplar as target as opposed to vice versa; prime and target related as opposed to prime and target unrelated; and the first six presentations as opposed to the last six presentations within each category, (i.e., the first vs. the second half of the study).
Second prime + mask trials. Prime + mask trials were conducted at the end of the study, to control for perceptual learning, sensory adaptation, and other sources of threshold drift. Hines et al. (1986) presented 12 words that had appeared on prime + mask + target trials, instructing the subjects that the words were familiar. We presented 12 words that had previously been presented on prime + mask + target trials, but, in addition, we also presented 12 unfamiliar but structurally similar words. Each set of 12 words was randomized separately for each subject, but the 12 unfamiliar words always followed the 12 familiar words. The 12 unfamiliar words were: $t a-$ ble, inch, hour, verb, cow, gold, orange, aunt, uncle, wool, green, and cup.

Hines et al. (1986) eliminated 8 subjects who identified 3 or more words in the series of 12 correctly. We included all 60 subjects in initial analyses, but we determined the consequences of excluding subjects who were able to identify words on prime + mask trials in separate analyses.

\section{Results}

First prime + mask trials. A total of 57 of the 60 subjects in the two experiments reached threshold at the minimum duration of $33.3 \mathrm{msec}$, and the remaining 3 reached threshold at $50 \mathrm{msec}$.

Prime + mask + target trials. Because the exposure duration for nearly all subjects was the minimum possible, we looked for possible ceiling effects within the identification accuracy distributions. Accuracies across all 48 trials ranged from $0 \%$ to $85.4 \%$. Although a total of six subjects identified all 12 first exemplar related primes and a total of three subjects identified all 12 fifth exemplar related primes, the two groups were disjoint; no subject identified all 12 first or fifth exemplar unrelated primes. We therefore concluded that the data were not greatly affected by ceiling effects. Moreover, only two subjects failed to identify at least one of the 48 primes, so that floor effects are also not a major concern.

In an analysis of variance, the two experiments were employed as one between-subjects variable. Knowledge of the pairs was a second between-subjects factor. Relatedness, order (first exemplar as prime/fifth exemplar as target or vice-versa), and presentation (first vs. second half) were the afforementioned within-subject variables. In all cases, the numbers of correct identifications were converted to percentages. Table 1 contains condition means and associated standard deviations.

The most important finding was that the accuracy of prime identification was better when a prime was followed by a related as opposed to an unrelated target $[F(1,56)$ $=83.77, M S \mathrm{e}=.0846, p<.01]$. Subjects who had learned the related pairs were more accurate than subjects who had not learned the pairs $[F(1,56)=18.30, M S e$ $=.3401, p<.01]$, and the effects of prime/target relatedness were greater for subjects in the knowledge group $[F(1,56)=12.65, M S e=.0846, p<.01]$.

These effects are portrayed in Figure 1. The main effect of relatedness illustrates that the target indeed does furnish cues that affect prime identification. We will define the priming effect as the difference between the ac- 
Table 1

Prime + Mask + Target Condition Means and Standard Deviations, Experiments 1 and 2

\begin{tabular}{|c|c|c|c|c|c|c|c|c|c|c|}
\hline \multirow[b]{4}{*}{ Condition } & \multirow[b]{4}{*}{ Condition } & \multirow[b]{4}{*}{ Order } & \multicolumn{8}{|c|}{ Experiment } \\
\hline & & & \multicolumn{4}{|c|}{1} & \multicolumn{4}{|c|}{2} \\
\hline & & & \multicolumn{2}{|c|}{ First Half } & \multicolumn{2}{|c|}{ Second Half } & \multicolumn{2}{|c|}{ First Half } & \multicolumn{2}{|c|}{ Second Hal } \\
\hline & & & $M$ & $S D$ & $M$ & $S D$ & $M$ & $S D$ & $M$ & $S D$ \\
\hline Knowledge & Related & $1-5$ & .82 & .13 & .91 & .11 & .73 & .23 & .81 & .25 \\
\hline Knowledge & Related & $5-1$ & .83 & .19 & .80 & .17 & .69 & .30 & .76 & .23 \\
\hline Knowledge & Unrelated & $1-5$ & .29 & .20 & .52 & .33 & .26 & .21 & .64 & .30 \\
\hline Knowledge & Unrelated & $5-1$ & .36 & .22 & .48 & .29 & .51 & .26 & .60 & .28 \\
\hline No-knowledge & Related & $1-5$ & .43 & .33 & .64 & .34 & .46 & .31 & .56 & .31 \\
\hline No-knowledge & Related & $5-1$ & .30 & .25 & .54 & .31 & .31 & .31 & .53 & .33 \\
\hline No-knowledge & Unrelated & $1-5$ & .26 & .26 & .38 & .34 & .27 & .31 & .39 & .31 \\
\hline No-knowledge & Unrelated & $5-1$ & .21 & .19 & .49 & .34 & .21 & .26 & .47 & .37 \\
\hline
\end{tabular}

Note-Each cell is based upon $n=15$.

curacy of prime identification with a related target and the accuracy of prime identification with an unrelated target. $^{2}$ This priming effect was $33.8 \%$ in the knowledge group but only $14.9 \%$ in the no-knowledge group (the priming effects within each of the two groups were both greater than zero when evaluated separately). The interaction thus denotes that the priming effect was enhanced by pretraining.

Relatedness and order is also interacted $[F(1,56)=$ $14.66, M S e=.0228, p<.01]$. This interaction is portrayed in Figure 2. It arose because the priming effect was $29.6 \%$ when the prime was the first exemplar of a category, but only $19.0 \%$ when the prime was the fifth exemplar.

Accuracy increased from $43.3 \%$ to $60.0 \%$ from the first to the second set of presentations $[F(1,56)=94.14, M S e$ $=.0314, p<.01]$. The knowledge $\times$ relatedness $\times$ presentation interaction was also significant $[F(1,56)=$ $5.95, M S e=.0398, p<.01]$. It reflects a large decrease in the priming effect in the knowledge group (from $41.4 \%$ to $25.8 \%$ across presentations), but a slight increase in the priming effect in the no-knowledge group (from $13.9 \%$ to $15.8 \%$ ).

There was a significant knowledge $\times$ order $\times$ presentation interaction $[F(1,56)=15.44, M S e=.0252, p<$ $.01]$. Identification accuracy increased more across presentations for first exemplar primes than for fifth exemplar primes in the knowledge group, but not in the noknowledge group. Both the increase in accuracy from first to second presentation and its interactions with knowledge and relatedness reflect perceptual learning.

Finally, the priming effect in Experiment 1 (29.9\%) was significantly greater than the priming effect in Experiment $2[18.8 \% ; F(1,56)=4.38, M S e=.0846, p<$ $.05]$, even though both effects were significantly greater than zero. This interaction is a manifestation of the intentional nature of prime identification in Experiment 1 as opposed to its incidental nature in Experiment 2.

Second prime + mask trials. In an analysis of variance of the second prime + mask trials, knowledge and experiments were employed as between-subjects variables and familiarity was employed as a within-subjects variable. The main effect of familiarity was highly significant
$[F(1,56)=307.51, M S e=.0557, p<.01]$. The interaction of condition and familiarity was also significant $[F(1,56)=6.35, M S e=.0557, p<.05]$. As Figure 3 illustrates, familiar words were seen quite readily, but unfamiliar words were not; and the difference was greater in the knowledge group than in the no-knowledge groups. Table 2 contains condition means and associated standard deviations.

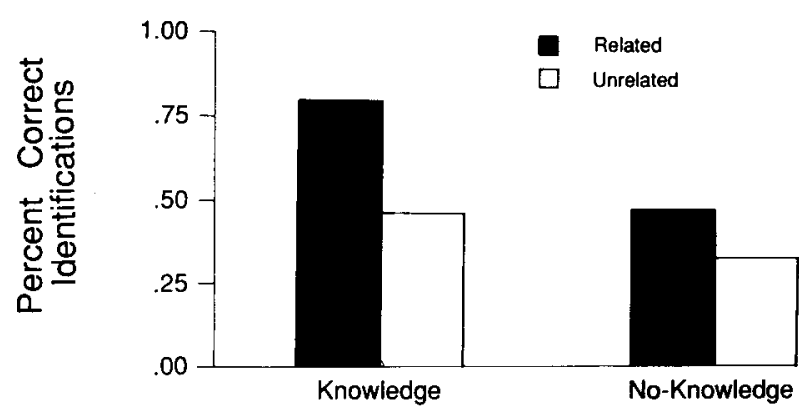

Experimental Condition

Figure 1. Identification accuracy as a function of knowledge and prime/target relatedness.

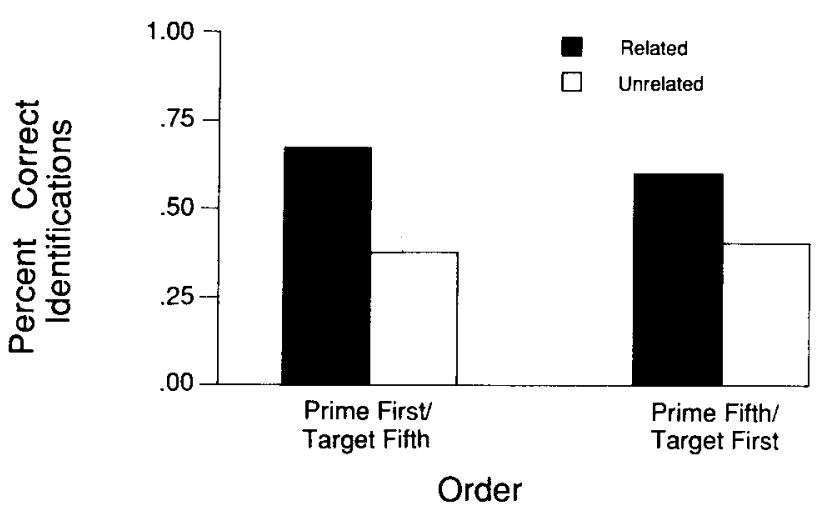

Figure 2. Identification accuracy as a function of prime/target relatedness and order (first exemplar as prime/fifth exemplar as target, or vice versa). 


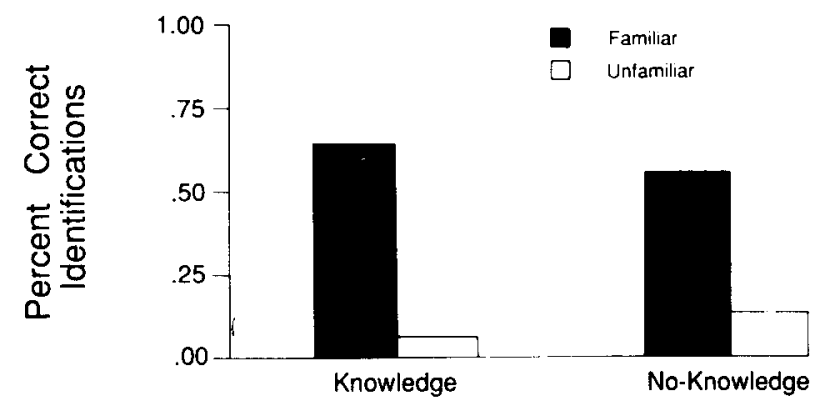

Experimental Condition

Figure 3. Identification accuracy in the second prime + mask condition, as a function of knowledge and familiarity.

Identification accuracy for familiar words was compared to identification accuracy on the second presentation of related first exemplar primes and related fifth exemplar primes. Knowledge and experiment were between-subjects variables. The main effect of type of prime was highly significant $[F(2,112)=9.18, M S e=$ $.0279, p<.01]$. The main effect of knowledge was also highly significant $[F(1,56)=10.96, M S e=.1522, p<$ $.01]$, as was the interaction of the two $[F(2,112)=5.37$, MSe $=.0279, p<.01]$. The essential finding is that subjects were less accurate in identifying primes presented alone than in the context of a related target. The effect was somewhat greater in the knowlege condition.

A parallel analysis was then conducted to compare the identification of familiar words with the identification of primes in unrelated word pairs. The only significant effect was that of type of prime $[F(2,112)=6.56, M S e=$ $.0396, p<.01]$. The accuracy in identifying familiar primes presented alone was greater than the accuracy in identifying primes followed by an unrelated target.

Collectively, the latter two analyses indicate that the target may be either facilitative or inhibitory, since the accuracy in identifying primes presented alone was intermediate in relation to the accuracy in identifying primes followed by related and unrelated targets. An examination of the distributions indicated that 2 subjects failed to identify any of the familiar words and that 1 identified all 12 , so there is no evidence of floor or ceiling effects having affected familiar words. A total of 26 subjects

Table 2

Second Prime + Mask Condition Means and Standard Deviations, Experiments 1 and 2

\begin{tabular}{|c|c|c|c|c|c|}
\hline \multirow[b]{3}{*}{ Condition } & \multirow[b]{3}{*}{ Word Type } & \multicolumn{4}{|c|}{ Experiment } \\
\hline & & \multicolumn{2}{|c|}{1} & \multicolumn{2}{|c|}{2} \\
\hline & & $M$ & $S D$ & $M$ & $S D$ \\
\hline Knowledge & Familiar & .66 & .21 & .62 & .25 \\
\hline Knowledge & Unfamiliar & .04 & .07 & .09 & .10 \\
\hline No-knowledge & Familiar & .56 & .30 & .57 & .26 \\
\hline No-knowledge & Unfamiliar & .09 & .09 & .18 & .19 \\
\hline
\end{tabular}

Note-Each cell is based upon $n=15$. failed to identify any of the unfamiliar words, and only 1 subject identified as many as 8 unfamiliar words. Consequently, floor effects seem to be present in these data.

Reexamination of prime + mask + target trials. Our final analyses were carried out to determine whether the relatedness effect was an artifact of our inability to reach threshold exposure duration. We repeated the analysis of variance of prime/target pairs, limiting analysis to 26 subjects, 16 in Experiment 1 and 10 in Experiment 2, who failed to identify any unfamiliar primes.

The relatedness effect in this subgroup was $.35[F(1,22)$ $=111.80, M S e=.0577, p<.001]$, and therefore slightly larger than the relatedness effect $(.24)$ inferred from the full sample for the two experiments taken collectively. The relatedness effects in the two experiments taken individually were .44 and $.20[F(1,14)=98.32$, $M S e=.0645, p<.001$, and $F(1,8)=18.11, M S e=$ $.0460, p<.01]$, as compared to .30 and .19 for the full sample. Despite the loss in power, all major effects (including the main effect of relatedness) that were significant in the full sample were also significant for subjects who met a rigorous definition of the term "subliminal." Our results are therefore not an artifact of subjects for whom the primes were visible.

\section{EXPERIMENT 3}

Thus far, the effects of target-induced context had been demonstrated in an identification task in which the subjects were not provided with information about possible response alternatives. These effects were obtained for subjects who did not identify any of the unfamiliar stimuli in the second prime + target condition and thus met a rigorous definition of "subliminal."

The purpose of Experiment 3 was to see if parallel effects could be obtained in a recognition task where subjects would have access to a set of response alternatives. There was a theoretical goal beyond that of simply extending findings obtained in an identification task. One possible mechanism for the effects of Experiments 1 and 2 was that the sole effect of the target was to reduce the number of response alternatives. If so, the target would not affect recognition performance because the ensemble of responses would serve that role more effectively. A second possibility was that the prime and target interacted so as to enhance other processing mechanisms, perhaps including those at a perceptual level. If that was the case, relevant targets would be effective in both recognition and identification tasks, though not necessarily to the same extent.

In addition to studying not only the identification but also the recognition task, we changed the stimulus materials to eliminate one troublesome feature in Experiments 1 and 2 and in Hines et al. (1986): the increasing familiarity with individual words that arises from their repetition. No word in Experiment 3 appeared more than once. 


\section{Method}

Subjects. Fifty-four subjects were obtained from the same source as the subjects employed in Experiment 1.

Stimuli and Apparatus. Two parallel lists were constructed, each consisting of 32 groups of four words. One word in each group served as the prime and therefore as a response alternative in the recognition test. A second word served as the target. A third word was structurally similar to the prime, and the fourth word was structurally dissimilar. The latter two words plus the prime constituted the response alternatives. Half of the prime/target pairings (16) on each list were related, and half were unrelated.

For example, WINE served as a prime on one of the lists, BEER served as a related target, and WINE, MINE, and NECK served as the response alternatives. Likewise, BOAT served as prime on that same list, MINT served as an unrelated target, and BOAT, GOAT, and FLEA served as the response alternatives. The two lists were related in that a given prime was paired with a related target on one list and with an unrelated target on the other list, the three response alternatives being the same on both lists. Consequently. WINE was paired with the unrelated target DESK, and BOAT was paired with the related target SHIP, on the second list. Each subject received only one of the two lists. The stimulus lists are available upon request from the authors.

The remaining aspects of the stimuli and apparatus (e.g., luminances) were identical to those in Experiments 1 and 2 .

Prime + mask + target trials. The procedure followed for all subjects parallelled the no-knowledge condition of Experiments 1 and 2. Consequently, there was no equivalent to the learning of related pairs. Instead, half the subjects were assigned at random to the identification condition. Their task and the stimulus sequence were identical to those in Experiment 1. The remaining subjects were assigned to the recognition condition, in which the three response alternatives associated with a prime appeared $750 \mathrm{msec}$ after offset of the target and remained on until the subject responded. In both cases, the subjects were given 5 practice trials followed by 32 test trials, half of which involved related prime/target pairings, while the remainder involved unrelated prime/target pairings. Consequently, task (identification vs. recognition) was a between-subjects variable, and prime/target relatedness was a within-subjects variable.

Second prime + mask trials. The final phase consisted of 12 identification trials and 12 recognition trials for all subjects, with order counterbalanced across subjects. Task was therefore a withinsubjects variable in this condition. All words used in this condition were novel, though structurally similar to those employed in the other condition.

\section{Results}

Initial prime + mask trials. A total of 51 out of 54 subjects reached threshold at the minimum duration of $33.3 \mathrm{msec}$. The remainder reached threshold at $50 \mathrm{msec}$.

Prime + mask + target trials. A preliminary analysis again failed to provide evidence for any ceiling or floor effect.

In an analysis of variance of the percentage of correct responses, task was employed as a between-subjects variable, and prime/target relatedness as a within-subjects variable. Not surprisingly, accuracy was higher in the recognition task $(M=.56)$ than in the identification task $[M=.21 ; F(1,52)=55.33, M S e=.0596, p<.001]$. Of greater importance was the significance of the prime/target relatedness effect in which accuracy for related pairs $(M=.49)$ was greater than accuracy for unrelated pairs $[M=.28 ; F(1,52)=1.73, M S e=.0270]$.
Table 3

Prime + Target + Mask Condition Means and Standard Deviations, Experiment 3

\begin{tabular}{clll}
\hline \multicolumn{1}{c}{ Task } & Condition & $M$ & $S D$ \\
\hline Identification & Related & .33 & .23 \\
Identification & Unrelated & .08 & .10 \\
Recognition & Related & .64 & .24 \\
Recognition & Unrelated & .47 & .22 \\
\hline
\end{tabular}

Note-Each cell is based on $n=27$.

To accept the null hypothesis that there is the same magnitude of relatedness effect in identification and recognition (.21) requires one to assume that the metrics are the same in the two cases. We do not need that strong an assumption to note that there is just as much evidence for a relatedness effect when subjects are given the response alternatives as when they are not.

The condition means and associated standard deviations are presented in Table 3. Analyses of error patterns, as, for example, in the choice of structurally similar as opposed to dissimilar responses, failed to add to the results. Specifically, the subjects chose the structurally similar incorrect response with essentially the same frequency on related and unrelated trials.

Second prime + mask trials. The analysis of variance of the second prime + mask trials included task as a withinsubjects variable and prime + mask + target task (also identification vs. recognition) as a between-subjects variable. The only significant effect was task; it is again not surprising that presenting the alternatives led to a greater accuracy $[M=.19 ; F(1,52)=2.19, M S e=.0604$, $p<.0011$. The condition to which the subjects were previously assigned had no effect. The condition means and associated standard deviations are also presented in Table 4.

Reanalysis of prime +mask + target trials. The fact that recognition accuracy $(M=.47)$ in the second prime + mask condition was well above the chance value of .33 meant that the stimuli were not truly subthreshold for the sample as a whole. We therefore went back and employed exclusionary procedures, as in Experiments 1 and 2, to determine the relatedness effect among the subjects who met various criteria for not having seen the stimuli in the second prime + mask condition.

The relatedness effect among 13 subjects who failed to identify any stimuli on the second prime + mask identification task was .12, as opposed to .21 for subjects in general. The effect was not significantly different from

Table 4

Second Prime + Mask Condition Means and Standard Deviations, Experiment 3

\begin{tabular}{llll}
\hline $\begin{array}{c}\text { Prime + Mask } \\
\text { +Target Condition }\end{array}$ & $\begin{array}{c}\text { Second Prime } \\
\text { +Mask Condition }\end{array}$ & $M$ & $S D$ \\
\hline Recognition & Recognition & .41 & .24 \\
Recognition & Recall & .23 & .21 \\
Recall & Recognition & .51 & .23 \\
Recall & Recall & .18 & .19 \\
\hline
\end{tabular}

Note-Each cell is based upon $n=27$. 
zero $[F(1,11)=2.97, M S e=.0502]$, and it had approximately the same magnitude in the identification (.10) and in the recognition task (.14). Consequently, the relatedness $\times$ task interaction was not significant. The relatedness effect was, however, significant among the 23 subjects who achieved at most one correct identification, which includes the 13 who had failed to identify any. The mean effect was $.14[F(1,21)=6.92, M S e=.0378, p<$ $.05]$. The effect had approximately the same magnitude in the identification task (.10) and the recognition task (.14). Thus, the relatedness $\times$ task interaction was nonsignificant $[F(1,11)=.05]$. The relatedness effect of .16 was also significant among 21 subjects with 4 or fewer correct recognitions, who were therefore at chance $[F(1,19)=12.49, M S e=.0315, p<.01]$. The magnitude of the relatedness effect was identical for subjects who had previously participated in an identification and a recognition task. Consequently, the relatedness $\times$ task interaction was zero, within rounding error. As was the case in Experiments 1 and 2, the results for subjects as a whole are not an artifact of subjects for whom the stimuli were not subliminal, regardless of whether an identification or recognition criterion is used.

\section{DISCUSSION}

Our primary findings were: (1) that the identification of unfamiliar stimuli presented alone was very poor; (2) that structurally similar words were accurately identified when relevant context was present in that related targets facilitated prime identification; (3) that unrelated targets inhibited prime identification; (4) that parallel findings were obtained with both an identification and a recognition criterion; and (5) that the results were not an artifact of our failure to have reached threshold.

Finding 1 is consistent with Marcel's findings. However, findings 2 and 3 illustrate that his effect does not require any "unconscious" mechanisms; it seems more closely related to the consequences of poststimulus cuing. Finding 4 also indicates that the target does more than simply restrict the number of response alternatives.

Collectively, these results are consistent with the view that prime and target mutually interact. At least one model of how priming affects retrieval, which is not concerned with Marcel's effect, explicitly treats prime and target as a compound event whose components may interact (Ratcliff \& McKoon, 1988). This model may be contrasted with the notion of spreading semantic activation (Neely, 1977; Posner \& Snyder, 1975).

The substantial effects of pretraining that we found, but that Hines et al. (1986) did not, do not really suggest an inconsistency, since we were concerned with responses to primes and they were concerned with responses to targets. A more serious inconsistency involves the high level of identification that we found for familiar primes presented alone. Perhaps our display differed in some critical manner from theirs-from their use of reverse video, for example. As previously noted, they did not present certain details such as stimulus size or luminance.

Unlike Hines et al. (1986), we found familiarity (frequency of presentation) to have substantial effects upon identification. These effects include the role of familiarity in both the identification of primes presented alone and the improvements in identifying prime/target pairings between the two presentations. Our findings are wellprecedented. Indeed, discussion of the alternative mechanisms underlying the role of familiarity (frequency) in perception was once the topic of vigorous inquiry. Broadbent (1967) has exquisitely summarized various models of the effect.

Our main conclusion is that inability to perceive a prime + mask sequence does not adequately define the "subliminal" nature of the prime. Just as exposure duration thresholds for semantic (or any other) material depend upon physical factors such as contrast and luminance, these thresholds also depend upon semantic context. No one would refer to the accurate perception of stimuli presented at, say, $10 \mathrm{msec}$ and $200 \mathrm{~cd} / \mathrm{m}^{2}$ as "subliminal" just because these same stimuli could not be seen at $10 \mathrm{msec}$ and $50 \mathrm{~cd} / \mathrm{m}^{2}$. In the same sense, it seems inappropriate to refer to the accurate perception of a word presented with a facilitative context as "subliminal" just because that word could not be perceived in the absence of context. The fact that an event does not contain sufficient information to yield a correct recognition or identification response does not rule out the possibility that its traces may interact with another event and thereby become adequate. The minimum requirement for definition of a stimulus as "subliminal" is that there must be a failure to perceive the prime when it is presented in context.

One way to implement such a control is to use a concurrent response paradigm (Eriksen, 1960), in which the subjects respond to primes and targets separately. Carr et al. (1982; see also McCauley et al., 1980) ran such a condition and noted that target naming latencies were actually longer when the prime was correctly identified as compared to when it was not. They attributed this difference to interference between retrieval operations when there was enough information to identify the prime. On the other hand, Dark (1988) has found that related primes produce facilitation only when they are correctly reported. She concluded that primes that undergo sufficient analysis to influence processing of the target also can be consciously reported. This would eliminate a simple interpretation of the origins of "subliminal" semantic priming. Our results are consistent with hers, which she has since replicated (Dark \& Benson, 1988). Briand, den Heyer, and Dannebring (in press) have obtained similar results. However, Fishler and Goodman (1978) found facilitation only for unreported primes.

It would be premature to attempt to reconcile these diverse results. Nonetheless, all explanations of priming assume that some form of interaction takes place when 
prime and target are processed. It is therefore important to consider the ways in which the two stimuli may interact. At least two contrasting interactions are likely present. Garner and Morton (1969; see also Ashby \& Townsend, 1986) note why one would expect a state correlation to arise because the subject's sensitivity varies over trials. On high sensitivity trials, both prime and target would be perceived more readily, and on low sensitivity trials, both would be perceived less readily, leading to a positive correlation between perceiving the two stimuli over trials. Conversely, limited capacity effects (Kahneman, 1973) would divert resources from the target while the prime is attended to and vice versa, to yield a negative process correlation. The two effects need not be of the same magnitude or be independent of semantic relatedness. Differences in the magnitudes of these correlations could also underlie the differences in types of errors for unmasked, energy masked, and pattern masked primes (Marcel, 1980). The essential point is to avoid treating "consciousness" of the prime as if it were a dichotomy (Eriksen, 1960).

One might additionally argue that the use of a mask "interrupts" processing of the prime. Of course, whether this is the case, or whether the effect is one of integration, remains an unresolved issue. Even if we grant the occurrence of interruptive mechanisms, fragmentary information about the prime may still remain to interact with the target, as in our DALLAS example. Only a complete "erasure" of the prime by the mask would prevent this interaction. Eriksen and Eriksen (1971) have offered a compelling critique of the erasure concept.

Recently, Cheesman and Merikle $(1984,1986)$ have suggested a distinction between subjective and objective thresholds that offers a possible role for subliminal effects. For example, they note that stimulus probability effects that are present above subjective threshold are not present below subjective threshold. But their distinction would not seem particularly useful here. Moreover, it seems quite plausible that had they used more than two intensities above their subjective threshold, they would have found more of a continuous relationship between intensity and the magnitude of the stimulus probability effect.

One definition of "subliminal" that we did not use involves the inability to detect the prime, and it may be that Merikle's (1982) objection may not hold universally. Greenwald, Klinger, Liu (in press) employed a forcedchoice detection task and obtained results that seem to support a "subliminal" position. Consequently, we cannot yet claim to have ruled out Marcel's position entirely. ${ }^{3}$ We do claim, however, to have noted an artifact, the failure to note the reciprocal nature of prime/target interactions, that bears many similarities to artifacts noted by the previous generation of researchers (Bernstein, Amundson, \& Schurman, 1973; Bernstein \& Eriksen, 1965; Eriksen, 1960). That is why we have devoted considerable attention to relevant studies from that era.

\section{REFERENCES}

AshBy, F. G., \& TownSEND, J. T. (1986). Varieties of perceptual independence. Psychological Bulletin, 93, 154-179.

Battig, W. F., Montague, W. E. (1969). Category norms for verbal items in 56 categories: A replication and extension of the Connecticut category norms. Journal of Experimental Psychology Monographs, Whole No. 80.

Bernstein, I. H, Amundson, V. E., Schurman, D. L. (1973). Metacontrast inferred from reaction time and verbal report: Replication and comment on the Fehrer-Biederman experiment. Journal of Experimental Psychology, 100, 195-201.

BerNSTEIN, I. H., \& ERIKSEN, C. W. (1965). Effects of "subliminal" prompting on paired-associate learning. Journal of Experimental Research in Personality, 1, 33-38.

Briand, K., den HeYer, K., Dannebring, G. L. (in press). Retroactive semantic priming in a lexical decision task. Quarterly Journal of Experimental Psychology.

BrondBENT, D. L. (1967). Word frequency effect and response bias. Psychological Review, 74, 1-15.

Carr, T. H., McCauley, C., Sperber, R. D., \& Parmelee, C. M. (1982). Words, pictures, and priming: On semantic activation, conscious identification, and the automaticity of information processing. Journal of Experimental Psychology: Human Perception \& Performance, 8, 757-777.

Cheesman, J., Merikle, P. M. (1984). Priming with and without awareness. Perception \& Psychophysics, 36, 387-395.

Cheesman, J., \& Merikle, P. M. (1986). Distinguishing conscious from unconscious perceptual processes. Canadian Journal of Psychology, 40, 343-367.

DARK, V. J. (1988). Semantic priming, prime reportability, and retroactive priming are interdependent. Memory \& Cognition, 16, 299-308.

DARK, V. J., \& BENSON, K. (1988). Semantic priming in a lexical decision task is not automatic. Paper presented at the Convention of the Midwestern Psychological Association, Chicago, IL.

Diener, D., \& SMEE, W. P. (1984). Apple tachistoscope. Behavior Research Methods, Instruments \& Computers, 16, 540-544.

ERIKSEN, C. W. (1960). Discrimination and leaming without awareness: A methodological survey and evaluation. Psychological Review, 67, 279-300.

ERIKSEN, C. W., \& ERIKSEN, B. A. (1971). Visual perceptual processing rates and backward and foward masking. Journal of Experimental Psychology, 89, 306-313.

Fishler, I., \& GoOdMAN, G. O. (1978). Latency of associated activation in memory. Journal of Experimental Psychology: Human Perception \& Performance, 4, 454-470.

Fowler, C. A., Wolford, G., Slade, R., \& Tessinary, L. (1981). Lexical access with and without awareness. Joumal of Experimental Psychology: General, 110, 341-362.

Garner, W. R., \& Morton, J. (1969). Perceptual independence: Definitions, models, and experimental paradigms. Psychological Bulletin, 72, 233-259.

Greenwald, A. G., \& Klinger, M. R. (1988). Semantic processing of dichoptically masked stimuli (Program of the Twenty-Ninth Annual Meeting of the Psychonomic Society). Bulletin of the Psychonomic Society, 26, 489. (Abstract)

Greenwald, A. G., KLinger, M. R., \& LiU, T. J. (in press). Unconscious processing of dichoptically masked words. Memory \& Cognition.

Hines, D., Czerwinski, M., SAwyer, P. K., DWyer, M. (1986). Automatic semantic priming: Effect of category level and word association level. Journal of Experimental Psychology: Human Perception \& Performance, 12, 370-379.

Holender, D. (1986). Semantic activation without conscious identification in dichotic listening, parafoveal vision, and visual masking: A survey and appraisal. Behavioral \& Brain Sciences, 9, 1-66.

Kahneman, D. (1973). Attention and effort. Englewood Cliffs, NJ: Prentice-Hall.

LAWRENCE, D. H. Coles, G. R. (1954). Accuracy of recognition 
with alternatives before and after the stimulus. Joumal of Experimental Psychology, 47, 208-214.

MARCEL, A. J. (1980). Conscious and preconscious recognition of polysemous words: Locating the selective effects of prior verbal context. In R. S. Nickerson (Ed.), Attention \& Performance VIII, (pp. 435457). Hillsdale, NJ: Erlbaum.

MARCEL, A. J. (1983a). Conscious and unconscious perception: An approach to the relations between phenomenal experience and perceptual processes. Cognitive Psychology, 15, 238-300.

MARCEL, A. J. (1983b). Conscious and unconscious perception: Experiments on visual masking and word recognition. Cognitive Psychology, 15, 197-237.

McCauley, C., Parmelee, C. M., Sperber, R. D., \& Carr, T. H. (1980). Early extraction of meaning from pictures and its relation to conscious identification. Joumal of Experimental Psychology: Human Perception \& Performance, 6, 265-276.

Merikle, P. M. (1982). Unconscious perception revisited. Perception \& Psychophysics, 31, 298-301.

Meyer, D. E., \& Schvaneveldt, R. W. (1971). Facilitation in recognizing pairs of words: Evidence of a dependence between retrieval operations. Joumal of Experimental Psychology, 90 , 227-234.

Natsoulas, T. (1967). What are perceptual reports all about? Psychological Bulletin, 67, 247-272.

NeELY, J. H. (1977). Semantic priming and retrieval from lexical memory: Roles of inhibitionless spreading of activation and limited capacity attention. Joumal of Experimental Psychology: General, 106, 226-254.

PIERCE, J. (1963). Some sources of artifact in studies of the tachistoscopic perception of words. Journal of Experimental Psychology, 66, 363-370.

Pollack, I., Rubenstein, H., \& Decker, L. (1959). Intelligibility of known and unknown message sets. Journal of the Acoustical Society of America, 31, 273-279.

PosNer, M. I., SNYDER, C. R. (1975). Attention and cognitive control. In R. Salso (Ed.), Information processing and cognition: The Loyola Symposium (pp. 55-82). Hillsdale, NJ: Erlbaum.

Purcell, D. G., Stewart, A. L., \& Stanovich, K. E. (1983). Another look at semantic priming without awareness. Perception \& Psychophysics, 34, 65-71.
RAtCliff, R., \& MCKoon, G. (1988). A retrieval theory of priming in memory. Psychological Review, 95, 385-408.

Rubenstein, H. C., \& Pollack, I. (1963). Word predictability and intelligibility. Journal of Verbal Learning \& Verbal Behavior, 2, 147-158.

SPERLING, G. (1960). The information available in brief visual presentations. Psychological Monographs, 74, (Whole No. 498).

SWETs, J. A., \& SEwall, S. T. (1963). Stimulus vs. response uncertainty in recognition. The Joumal of the Acoustical Society of America, 33, 1586-1592.

TulviNG, H. I., Gold, C. (1963). Stimulus information and information as determinants of tachistoscopic recognition of words. Journal of Experimental Psychology, 66, 319-327.

\section{NOTES}

1. It may be noted that the prime-mask trials involved in a two-stimulus sequence, whereas the prime + mask + target trials involved a threestimulus sequence. However, Purcell et al.'s (1983) comments do not apply, though they may apply to Hines et al. (1986). Their comments were directed against studies using reverse video or other situations in which flashes of light appear interspersed among dark intervals to affect adaptation. Our use of conventional video meant that the amount of light varied trivially within a trial so that adaptation was not affected.

2. A conceptually better definition would refer to the difference in identification accuracy between related and noninformative targets. We did not include noninformative targets on prime + mask + target trials because Hines et al. (1986) did not. We will, however, make comparisons of this form below, using data from the second prime + mask trials. The definition we used is simply an heuristic device to facilitate description.

3. As the present article was going to press, Greenwald and Klinger (1988) reported a failure to replicate Greenwald et al.'s (in press) results, possibly making the point moot.

(Manuscript received June 20, 1988; revision accepted for publication August 8, 1988.) 